\title{
Magnitude of Obstructed Labor and Associated Risk Factors among Mothers Come for Delivery Service in Adama Hospital Medical College, Oromia Regional State, Central Ethiopia
}

\author{
Asnakech Tadesse Gudina ${ }^{1}$, Tilaye Workneh Abebe ${ }^{2, *}$, Fikiru Abebe Gebremariam³, \\ Godana Jarso Guto ${ }^{4}$ \\ ${ }^{1}$ Department of Integrated Emergency Surgery and Obstetrics, Adama Hospital Medical College, Adama, Ethiopia \\ ${ }^{2}$ Department of Public Health, Adama Hospital Medical College, Adama, Ethiopia \\ ${ }^{3}$ Department of Obstetrics and Gynecology, Adama Hospital Medical College, Adama, Ethiopia \\ ${ }^{4}$ Department of Internal Medicine, Adama Hospital Medical College, Adama, Ethiopia
}

Email address:

tlife2002@gmail.com (T. W. Abebe)

${ }^{*}$ Corresponding author

To cite this article:

Asnakech Tadesse Gudina, Tilaye Workneh Abebe, Fikiru Abebe Gebremariam, Godana Jarso Guto. Magnitude of Obstructed Labor and Associated Risk Factors among Mothers Come for Delivery Service in Adama Hospital Medical College, Oromia Regional State, Central Ethiopia. Journal of Gynecology and Obstetrics. Vol. 4, No. 3, 2016, pp. 12-16. doi: 10.11648/j.jgo.20160403.11

Received: April 8, 2016; Accepted: April 20, 2016; Published: June 13, 2016

\begin{abstract}
Background: Obstructed labor is one of the common preventable causes of maternal and perinatal morbidity and mortality in developing countries. In the last decade among the top four causes of maternal mortality, obstructed labor /uterine rupture accounted for $36 \%$ of maternal mortality in Ethiopia. The aim of this study was to determine magnitude of obstructed labor and its associated risk factors among mothers come for delivery services in Adama Hospital Medical College from June 01 to July 30, 2015. Methods: Hospital-based cross-sectional study was employed. Descriptive statistics was used to summarize socio-demographic variables and characteristics of delivery. Using binary logistic regression analysis, Odds ratio and confidence interval were used to determine statistically associated risk factors with obstructed labor. Result: Of 384 deliveries during the study period $9.6 \%$ were obstructed labor cases. The causes of obstructed labor identified by clinician on mother medical record were CPD (54.1\%), mal-position (29.7\%) and mal-presentation 16.2\%). The independent predictors of obstructed labor using logistic regression analysis were duration of labor before arrival to the health facility greater than 24 hour (OR: $6.2 ; 95 \% \mathrm{CI}: 2.7,14.2), 4$ to 6 hours of travel to health facility (OR: 2.9; 95\%CI: 1.1, 10.6), malpresentation (OR: 6; 95\%CI: $1.7,12.8)$ and fetal weight greater than 4 Kilo gram (OR: 11.2; 95\%CI: 2.4, 13.2). Conclusion: magnitude of OL is comparatively higher, especially among mothers of low educational status. Socio-demographic and health system factors increase the risk of obstructed labor. As most of the risk factors associated with obstructed labor need long term exposure for the outcome to be seen, prevention need inter sectoral collaboration from education, agriculture, health and other social sectors.
\end{abstract}

Keywords: Risk Factors, Obstructed Labor, Risk Prevention

\section{Introduction}

Obstructed labor (OL) is the failure of descent of the fetal presenting part in the birth canal for mechanical reasons. Obstruction could be because of faults in maternal pelvis (abnormal shape or size) or the fetus (abnormally large, presenting abnormally, mal-positioned or congenitally abnormal). OL is an important cause of maternal death in communities where childhood under nutrition and early marriage are common that result in small pelvis and low access to health facilities [1].

There is varying magnitude of OL from as low as $1.3 \%$ a study done in Sudan to as high as $7 \%$ in a retrospective study 
done at Jima University specialized hospital, Ethiopia. The major cause of obstructed labor identified in different studies done in developing countries was cephalopelvic disproportion, and malpresentation/mal position [2, 3, 4].

Cephalo Pelvic Disproportion (CPD) stands out to be the most significant contributor of OL. Specific pelvic deformities such as those due to rickets or oseomalasia may be important in some areas but the commonest is generally pelvic contracture. This is closely related to impairment of growth by ill health and malnutrition in childhood and adolescent. The other important abuses of obstruction are mal-presentation such as impacted transverse lie, breech, brow and face presentation. Apart from bone abnormalities, soft tissue abnormalities are occasionally responsible for OL such as an unyielding stenosis of the cervix (vagina) and ovarian tumors impacted on the pelvis. OL may also occur in spite of a capacious pelvis if the fetus is abnormal. Common causes include hydrocephalus, monsters and locked twins $[5,6]$.

$\mathrm{OL}$ is not significant health problem in developed countries. In developing countries the main causes of obstructed labour include large or abnormally positioned baby, a small pelvis, and problems with the birth canal. Abnormal positioning includes shoulder dystocia (anterior shoulder does not pass easily below the pubic bone). The risk factors for a small pelvis include malnutrition and a lack of exposure to sunlight causing vitamin D deficiency [7]. Study done in South-Western Uganda reported the main causes of obstructed labor were CPD (63.3\%), malpresentation or malposition (36.4\%) and hydrocephalus $(0.3 \%)$. The risk of obstructed labor was with nulliparous status (AOR 1.47, 95\% CI: 1.22-1.78), having delivered once before (AOR 1.57, 95\% CI: 1.30-1.91) and age group 15-19 years (AOR 1.21, 95\% CI: 1.02-1.45). The risk for perinatal death as an adverse outcome was statistically significantly associated with grand multiparous status (AOR 1.89, 95\% CI: 1.113.22). Women who lacked paid employment were at increased risk of obstructed labor [8]. Similarly, study done in Northern Ethiopia reported that the most common cause of obstruction was cephalopelvic disproportion (64.9\%) followed by malposition/malpresentation (32.5\%) [9].

OL results ruptured uterus or puerperal infection that cause maternal mortality and asphyxia that causes perinatal mortality. Considerable maternal morbidity is associated with prolonged labor, since both post-partum hemorrhage (PPH) and infection are more common in women with long lasting labor which may be due to OL. Obstetric fistulas are longterm complications of OL (9). OL was identified as the direct cause of maternal death in $22 \%$ of the cases. Maternal complications of OL were ruptured uterus, puerperal sepsis, bladder injury, PPH and fistula [8].

$\mathrm{OL}$ is a major cause of maternal morbidity and mortality in low income countries and accounts for approximately $8 \%$ of maternal deaths globally [9]. Studies in low-income countries have reported that the prevalence of OL is between $2-8 \%$ of all institutional deliveries $[12,13]$. This may underestimate maternal deaths due to obstructed labor because it is rarely documented as a primary cause of death rather classified as, rather classified as sepsis, ruptured uterus or hemorrhage rather than the underlying cause [14].

$\mathrm{OL}$ is responsible for immediate and late complications which lead to high maternal mortality, still birth and early neonatal death. The immediate complications are like: postpartum hemorrhage (PPH) uterine rupture, intra and postpartum infection leading to peritonitis, sepsis, septic shock, fetal distress and death, and neonatal sepsis. The late complications are vesicovaginal fistula (VVF), recto-vaginal fistula (RVF), vagina stenosis and stricture, foot drops, contracture of joint and otitis pubis, perinatal asphyxia and mental retardation [5].

The magnitude and risk factors associated with OL was not studied in the study setting and the region as a whole. Thus, this particular study aimed to determine the magnitude and risk factors associated with OL which help to plan for better intervention of such problem in this particular study setting.

\section{Methods and Materials}

\subsection{Study Design and Setting}

Facility based cross sectional study design was employed to determine magnitude and risk factors associated with OL in Adama Hospital Medical College (AHMC), Oromia regional state, central Ethiopia. The current AHMC was established as missionary hospital in 1946. It was referral hospital in central Oromia regional state before four years and established as hospital based Medical College in 2011 by Oromia regional state.

The study was conducted from June 01 to July 30, 2015. All mothers who attended the delivery room of AHMC during the study period were included consecutively. Interviewer administered questionnaire was used to collect personal information from study participants. Data abstraction format was used to collect clinical information from respondent medical record which complements the primary data obtained from respondents. The data collection tools adopted from known similar study was pretested before the actual data collection activity. Interview tool was translated to local language accordingly. Trained data collectors were used to collect data and supervisors followed the data collection process and provided necessary correction immediately during data collection.

Ethical clearance was secured from AHMC Institutional Ethical Review Board. Informed oral consent was obtained for each study subject before data collection. Participation was on voluntary basis. Respondent information was kept confidential and used for the research purpose only.

\subsection{Sample size Determination}

Sample size was calculated using single population proportion formula considering 95\% level of significance, $5 \%$ margin of error and $50 \%$ proportion $(\mathrm{P})$ of obstructed labor for maximum sample size. Accordingly the total sample size will be, 
$\mathrm{N}=\frac{\left(\frac{\mathrm{z} \alpha}{2}\right)^{2} \mathrm{p}(1-\mathrm{p})}{\mathrm{d}^{2}}=\frac{(1.96)^{2}(0.5)(1-0.5)}{(0.05)^{2}}=384$ mothers

\subsection{Data Analysis}

After the collected data was edited and coded, it was entered into Epi Info Version 7 statistical program. Then, the data was exported to SPSS Version 20 for cleaning and analysis. Descriptive statistics was used to summarize sociodemographic and to estimate magnitude of OL. Binary logistic regression analysis was used to identify factors associated with OL. Both crude and adjusted odds ratio with $95 \%$ confidence interval was used to determine statistical association. P-value $<$ 0.05 was used to define statistical significance.

Dependent variables

$\mathrm{OL}$ - is a failure of descent of the fetal presenting part for mechanical reasons in spite of adequate uterine contractions

Independent variables:

CPD - is the disproportion between the sizes of the fetus relative to the mother and can be the cause of a protraction or arrest disorder

Mal-position - is abnormal relationship of the fetal presenting part to the maternal pelvis

Mal-presentation - is any fetal part other than vertex or the fetal part that is not directly overlies the pelvic inlet

Macrosomia - is growth beyond a specific threshold or fetal weight greater than $4000 \mathrm{~g}$

\section{Result}

\subsection{Socio-Demographic Characteristics of Respondents}

The respondent's socio-demographic characteristics were summarized in table 1 below. Three hundred eighty four delivery cases were included in this study. The mean age of the respondents was 25 years with standard deviation of 5 years. The majority, $302(78.65 \%)$ of the respondents were rural residents. The large majority, $93.2 \%$ of the respondents were married. Mothers who had no formal education account $18.2 \%$ of the respondents while $38.8 \%$ had completed at least high school. Slightly more than half, $52.9 \%$ of the respondents were house wife/dependent on their husband income for life.

Table 1. Socio demographic characteristics of respondents, June 01 to July 30, Adama, 2015

\begin{tabular}{|c|c|c|}
\hline Variables & & Number (\%) \\
\hline \multirow{3}{*}{$\begin{array}{l}\text { Age (in full } \\
\text { years) }\end{array}$} & $15-24$ & $186(48.4)$ \\
\hline & $25-34$ & $171(44.5)$ \\
\hline & $35-44$ & $27(7)$ \\
\hline \multirow{2}{*}{ Residence } & Rural & $302(78.6)$ \\
\hline & Urban & $82(21.4)$ \\
\hline \multirow{3}{*}{ Marital status } & Unmarried & $21(5.5)$ \\
\hline & Married & $358(93.2)$ \\
\hline & Divorced & $5(1.3)$ \\
\hline \multirow{3}{*}{ Religion } & Orthodox & $198(51.6)$ \\
\hline & Muslim & $120(31.3)$ \\
\hline & Protestant & $66(17.2)$ \\
\hline Ethnicity & Oromo & $236(61.5)$ \\
\hline
\end{tabular}

\begin{tabular}{lll}
\hline Variables & & Number (\%) \\
\hline & Amhara & $83(21.6)$ \\
& Tigre & $13(3.4)$ \\
& Gurage & $17(4.4)$ \\
& others & $35(9.1)$ \\
Education & Non educated & $70(18.2)$ \\
& Elementary & $165(43.0)$ \\
& High school and above & $149(38.8)$ \\
& House wife & $203(52.9)$ \\
& Civil employee & $38(9.9)$ \\
& Merchant & $40(10.4)$ \\
& Farmer & $74(19.3)$ \\
& Others & $29(7.6)$ \\
& Total & $384(100)$ \\
\hline
\end{tabular}

\subsection{Ante Natal Care (ANC) Follow up and Previous Delivery History}

Table 2 below shows ANC follow up and delivery related information. Majority of the respondents, $91.3 \%$ had at least one ANC follow up. One hundred forty eight (38.5\%) of the participant were delivered their previous baby in health facility. The majority $(68.5 \%)$ of the respondent were referred from nearby clinic/health center.

Table 2. ANC follow up and delivery related information among mothers delivered AHMC from June 01 to July 30, 2015.

\begin{tabular}{lll}
\hline Health service utilization & & Number (\%) \\
\hline \multirow{2}{*}{ ANC follow up } & Yes & $352(91.3)$ \\
& No & $32(8.3)$ \\
Previous place of delivery & Home & $236(61.5)$ \\
& Health institution & $148(38.5)$ \\
& Prolonged labor & $17(4.4)$ \\
Reason to come AHMC & Referred & $263(68.5)$ \\
& Want to deliver at H/L & $88(22.9)$ \\
& Appointment & $16(4.2)$ \\
\hline
\end{tabular}

\subsection{Magnitude of Obstructed Labour}

There were $37(9.6 \%)$ cases of OL among 384 participants included in the study of which $34(91.9 \%)$ were referred from nearby health facilities. As reported on mothers' card by clinicians, CPD was identified as a cause of OL in 20(54.1\%) of the cases. Mal-position and mal-presentation was the cause of OL in $11(29.7 \%)$ and $6(16.2 \%)$ of the cases respectively.

\subsection{Predictors of Obstructed Labor}

Of variables significantly associated in the univariate analysis and included in multivariate model, occupation, time of transportation, and time of intervention, were dropped from the final model. Duration of labor before arrival to health facility greater than 24 hour had statistically significant association with OL (OR: 6.2; 95\%CI: $2.7,14.2$ ). Longer time to reach health facility, 4 to 6 hours of travel to health facility had statistically significant association with OL (OR: 2.9; 95\%CI: 1.1, 10.6). Similarly, malpresentation (OR: 6; 95\%CI: $1.7,12.8$ ) and fetal weight greater than 4 Kilo gram (OR: 11.2; 95\%CI: 2.4, 13.2) had statistically significant association with OL (Table 3). 
Table 3. Predictors of OL among mothers delivered in AHMC from June 01 to July 30, 2015.

\begin{tabular}{|c|c|c|c|c|c|}
\hline \multirow{2}{*}{ Variables } & & \multicolumn{2}{|l|}{ Obstructed labor } & \multirow{2}{*}{ Crude OR(95\%CI) } & \multirow{2}{*}{ Adjusted OR(95\%CI) } \\
\hline & & Yes Number (\%) & No Number (\%) & & \\
\hline \multirow{5}{*}{ Occupation } & House wife & $17(45.9)$ & $186(53.6)$ & $3.4(0.4,26.2)$ & $2.5(0.3,22.4)$ \\
\hline & Employee & $1(2.7)$ & $37(10.7)$ & 1.0 & 1.0 \\
\hline & Merchant & $2(5.4)$ & $38(11)$ & $1.95(0.2,22.4)$ & $1.0(0.1,14.2)$ \\
\hline & Farmers & $15(40.5)$ & $59(17)$ & $9.4(1.2,74.22)$ & $2.3(0.2,22.4)$ \\
\hline & others & $2(5.4)$ & $27(7.8)$ & $6.73(0.6,81.4)$ & $7.7(0.5,118.8)$ \\
\hline Duration of labor before arrival to & $\leq 24 \mathrm{hrs}$ & $12(32.4)$ & $278(80.1)$ & 1.0 & 1.0 \\
\hline \multirow[t]{2}{*}{ AHMC/Facility } & $>24 \mathrm{hrs}$ & $25(67.6)$ & $69(19.9)$ & $8.4(4.0,17.5)$ & $6.2(2.7,14.2) *$ \\
\hline & $<2 \mathrm{hrs}$ & $21(56.8)$ & $229(66)$ & 1.0 & 1.0 \\
\hline Time taken to reach & $2-4 \mathrm{hrs}$ & $7(18.9)$ & $88(25.4)$ & $0.87(0.4,2.1)$ & $0.5(0.2,1.3)$ \\
\hline \multirow[t]{2}{*}{ AHMC/facility } & $4-6 \mathrm{hrs}$ & $6(16.2)$ & $21(6.1)$ & $3.1(1.3,8.6)$ & $2.9(1.1,10.6)$ \\
\hline & $>6 \mathrm{hrs}$ & $3(8.1)$ & $9(2.6)$ & $3.6(0.9,14.5)$ & $0.7(0.1,4.2)$ \\
\hline \multirow{2}{*}{ Time of delivery intervention/ } & $<6 \mathrm{hrs}$ & $34(91.9)$ & $265(76.4)$ & 1.0 & 1.0 \\
\hline & $\geq 6 \mathrm{hrs}$ & $3(8.1)$ & $82(23.6)$ & $3.51(1.1,11.72)$ & $0.3(0.1,1.3)$ \\
\hline \multirow{3}{*}{ Mal-presentation } & yes & $6(16.2)$ & $14(4)$ & $4.6(1.7,10.8)$ & $6.0(1.7,12.8) *$ \\
\hline & No & $31(83.5)$ & $333(96)$ & 1.0 & 1.0 \\
\hline & $<2.5 \mathrm{~kg}$ & $2(5.4)$ & $37(10.7)$ & 1.0 & 1.0 \\
\hline \multirow[t]{2}{*}{ Fetal weight } & $2.5-3.9 \mathrm{~kg}$ & $23(62.0)$ & $289(83.3)$ & $1.5(0.3,6.5)$ & $1.8(0.3,9.2)$ \\
\hline & $\geq 4 \mathrm{~kg}$ & $12(32.4)$ & $21(6.1)$ & $8.5(2.2,12.8)$ & $11.4(2.4,13.2) *$ \\
\hline
\end{tabular}

*indicates significant at $95 \% \mathrm{CI}, \mathrm{P}<0.05$

\section{Discussion}

Worldwide, up to $15 \%$ of pregnancies might develop a risk of complications and these usually come unpredicted and unexpected. Whether the outcome will be threatening to the life of the mother and/ or baby mainly depends on the availability of proper obstetric health care provision [15]. Obstructed labor is dangerous pregnancy complication, which has almost disappeared from the western world, but is still one of the leading causes of maternal death in developing countries [9]. Obstructed labor contributed 4.1\% (range $0-10.3 \%$ ) to all maternal deaths in Africa; for Asia to $9.4 \% \quad(0-12 \%)$ and $13.4 \%$ (range $0-38.9 \%$ ) for Latin America and Caribbean [16].

In this study the magnitude of obstructed labor is $9.6 \%$ which consistent with other study done in developing countries. Study done in Jima University hospital western part of Ethiopia reported $12.2 \%$ OL cases. Similarly, study done in Western Uganda and Nigeria reported $10 \%$ of OL cases $[14,17,18]$. But, the finding of this study is higher than study done in Adigrat Hospital (3.3\%) in Northern Ethiopia [9]. This is may be the study health facility is referral center and geographical accessibility which increase referral cases and the finding also agree with this assumption because $91.9 \%$ of the cases are referral.

As clinicians reported the cause of OL on mothers' medical record, 20(54.1\%) of OL cases were due to CPD. Mal-position and mal-presentation was the cause of OL in $11(29.7 \%)$ and $6(16.2 \%)$ of the cases respectively. The finding is consistent with the study done in similar settings $[9,14,16]$.

The finding of this study showed $54 \%$ of OL were among mothers with education status less than grade five, even though there is no statistical association. This is may be these mothers had less income/resource and nutrition background which increase the risks of obstructed labour. Other studies done in similar setting reported similar scenario, in which socio-demographic factors are important risks for OL [9].

Duration of labor before arrival to health facility greater than 24 hour is statistically significant association with obstructed labour. Longer time to reach health facility, 4 to 6 hours of travel to health had statistically significant association with OL. This is may be most rural mothers are had low health seeking behavior and low resource for health service cost which delay decision. On the other hand, these mothers may have increased OL risk in relation to educational status, distance, nutritional habit and knowledge, and other factors.

This study indicates that being macrosomic (fetal weight $\geq 4 \mathrm{~kg}$ ) has 11.2 times higher risk of developing OL than normal weight. The presentation of higher birth weights makes sense: the bigger the baby the easier the passage might be inadequate. Macrosomic fetus increases the risk of maternal as well as fetal complication.

\section{Conclusion}

In this study, the magnitude of OL is comparatively higher than Western and even some of developing countries. It is higher among respondents of low educational status. Long duration of labor before arrival to health facility, long hour of travel to health facility and other health system factors increase the risk of OL. Most of the cases are preventable by proper health care system using trained health workers, together with early referral of high-risk patients, educating mothers and proper nutrition during childhood and adolescence. This study may provide baseline information for policy maker and health planner about socio-demographic and other factors associated with OL. As most of the risk factors associated with OL need long term exposure for the outcome to be seen, prevention need inter sectoral 
collaboration action from education, agriculture, health and other social sectors.

\section{Author's Contributions}

All authors were involved in conception, design, analysis, interpretation and write up of this study. All the authors read and approved the final content of the manuscript.

\section{Acknowledgements}

The Authors acknowledge all the study participants and data collectors. The authors also acknowledge AHMC for their support.

\section{References}

[1] Arrow S, Hamlin C, Wall L. Obstructed labor injury complex: obstetric fistula formation and the multifaceted morbidity of maternal birth trauma in the developing world. Obstet Gynecol Surv. 1996; 51(9): 568-74.

[2] Ozumba C, Uchegbu H. Incidence and management of obstructed labor in Eastern Nigeria. Aust N Z J Obstet Gynaecol. 1991; 31(3): 213-6.

[3] Dafallah E, Ambago J, El-Agib F. Obstructed labor in a teaching hospital in Sudan. Saudi Med J. 2003; 24(10): 11024.

[4] Chabra S, Gandhi D, Jaiswal M. Obstructed labor - a preventable entity. J Obstet Gynaecol. 2000; 20(2): 151-3.

[5] Miane M. Fraser, Margaret A. Cooper Mayle's midwife text book. Elseveir: Churchil Livingstone; 2008; 545-6.

[6] Gaym A. Obstructed labor at district hospitals. Ethiop Med J, 2002; 40(1): 11-8.
[7] WHO: Education material for teachers of midwifery: midwifery education modules (2nd ed.). Geneva, Switzerland, 2008; pp. 38-44. ISBN 9789241546669.

[8] Kabakyenga, JK, Ostergren, PO, Turyakira, E, Mukasa, PK \& Odberg-Petterson, K. Individual and health facility factors and the risk for obstructed labor and its adverse outcomes in south-western Uganda. Biomed Central Pregnancy and Childbirth. 2011b; 11(73): 1-10.

[9] Gessessew, A \& Mesfin, M. Obstructed labor in Adigrat Zonal Hospital, Tigray region, Ethiopia. Ethiopian Journal of Health Development. 2003; 17(3): 175-180.

[10] Dolea, C \& AbouZahr, C. The global burden of obstructed labor in the year 2000. Geneva: WHO, 2003.

[11] WHO: The World health report: make every mother and child count. Geneva: World Health Organization; 2005, 230.

[12] Nwogu-Ikojo EE, Nweze SO, Ezegwui HU: Obstructed labor in Enugu, Nigeria. J Obstet Gynaecol. 2008; 28(6): 596-599.

[13] Ali AA, Adam I. Maternal and perinatal outcomes of obstructed labor in Kassala hospital, Sudan. J Obstet Gynaecol. 2010; 30(4): 376-377.

[14] Kabakyenga et al. obstructed labor and birth preparedness. 2011; 11:73 http://www.biomedcentral.com/ 1471-2393/11/73

[15] WHO, UNICEF, UNFPA and the World Bank. Maternal Mortality Estimates in 2005. Geneva: WHO, 2007a.

[16] Kahn, K, Wojdyla, D, Say, L, Gülmezoglu, M \& Van Look, P. 2006. WHO analysis of causes of maternal death: a systematic review. Lancet 367: 1066-1074.

[17] Shimelis, F, Hailemariam, S \& Fessahaye, A. Incidence causes and outcome of obstructed labor in Jima University specialized hospital. Ethiopian Journal of Health Science. 2010; (20)3: 145-151.

[18] Yakasai IA, Gaya AS. Maternal and fetal outcomes in patients with eclampsia in Murtala Muhammed specialist Hospital Kano Nigeria. Annals Afri. Med. 2011; 10(4): 305-309. 